\title{
Confidence ratings in recall paired-associates learning'
}

MILTON D. SUBOSKI, BRUCE A. PAPPAS AND D. J. MURRAY

QUEEN'S UNIVERSITY, KINGSTON, ONTARIO

Having $S$ s rate their confidence in the correctness of their responses in a simple verbal learning task yielded results consistent with the hypothesis that confidence ratings reflect the strength of the learned association between stimuli and responses. The results further suggest a continuum of states of associative strength rather than just two: learned and unlearned.

The recent application of statistical decision theory to psychophysics has demonstrated a continuity in stimulus detection quite contrary to threshold hypotheses (Swets, Tanner, \& Birdsall, 1961). It has further been shown that ratings of confidence of decisions can serve as accurate indices of the criteria used in making decisions about sensory states (Egan, Schulman, \& Greenberg, 1959). Egan (1958) has extended signal detection techniques to analysis of recognition memory. Murdock (1965), using both a recognition test and confidence ratings, concluded that short-term recognition memory "seems more like the continuous process postulated by signal-detection theory with the individual capable of varying his criterion over a wide range" ( $p .446)$ rather than like the "high-threshold" process postulated by Underwood \& Keppel (1962)。 He points out, however, that the Underwood and Keppel hypothesis was originally formulated for recall.

The present study extends the use of confidence ratings to the recall paradigm in an effort to examine the hypothesis that the ratings reflect the underlying associative strength between stimuli and responses. Also examined is the question of whether there is a continuum of associative strength or merely two states: learned and unlearned.

Method

Twenty Ss learned 10 lists, each consisting of a random pairing of the letters A-J as stimulus items and the numbers $0-9$ as responses. The simplicity of the items was intended to confine learning to stimulus-response associations and to eliminate the need to learn what items were being used.

Each list of stimulus-response pairs was presented three times at a $4 \mathrm{sec}$.exposure rate by a "flash-card" technique. Each presentation was followed by a test in which Ss worked through a 10 page booklet at a $7 \mathrm{sec}$. per page rate. Each page contained a stimulus letter, a space to write in the response number, and a $5 \mathrm{~cm}$ "confidence" line which had the left end labelled "guess" and the right end labelled "sure." Ss were instructed to draw a vertical line across the confidence line to indicate their degree of confidence in the correctness of their written response. The order of lists and the order of pairs within a list for the three presentations were constant for all Ss. The order of stimulus items within booklets was random for each test for each S.

Data were obtained in two group sessions, one of 14 Ss, the other of 6 . Lack of substantial differences between the two sessions resulted in combining of the data. Results

Confidence ratings were classified into five equal categories of $1 \mathrm{~cm}$ lengths on the confidence line. Proportion of correct responses, $\mathrm{p}(\mathrm{c})$, for each confidence category, pooled across Ss and test trials, are shown in Fig. 1. The increase in $p(c)$ with increasing confidence does not appear to be an artifact of averaging over Ss, since four sign tests performed on individual $S$ data indicated that $p(c)$ is reliably greater $(p ' s<.05)$ for the higher of each pair of adjacent categories. The individual curves for the three test trials, pooled across Ss (not shown) are remarkably similar to the mean shown in Fig. 1, with .064 being the largest difference between any two curves. This invariance in $p(c)$ for the confidence categories across test trials rules out the possibility that the increase in $\mathrm{p}(\mathrm{c})$ across categories is simply a result of the simultaneous increase in the use of the higher categories and overall $\mathrm{p}$ (c)(from .599 to .792 to .869) across the three test trials.

The lower curve of Fig. 1 indicates that the proportion of shifts from correct to incorrect on two successive

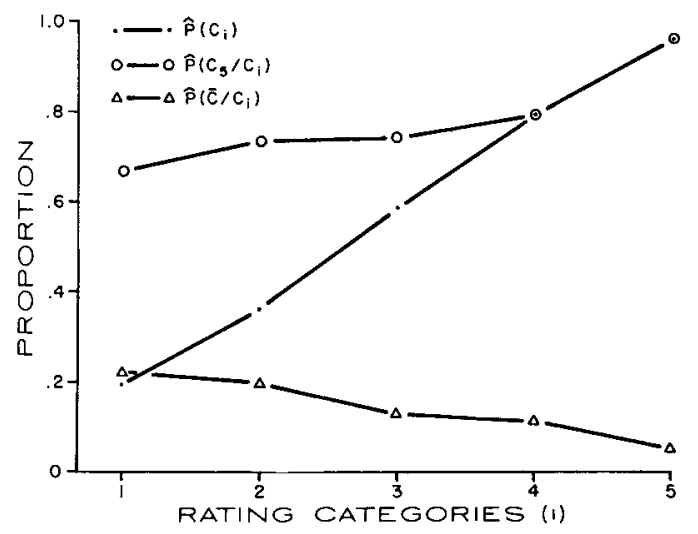

Fig. 1. Upper curve: Proportion of shifts to the highest confidence on the second trial, given correct on both, for each confidence category on the first.

Middle curve: Proportion of correct responses for each category.

Lower curve: Proportion of shifts from correct to incorrect for each of the categories on the first trial. 
test trials is a monotonically decreasing function of the confidence category on the first. The proportion of shifts in the lower confidence categories are substantially higher than the .077 overall proportion of such shifts.

The upper curve of Fig. 1 shows that the proportion of shifts to the highest confidence, given correct on both trials (C-C) is an increasing function of the confidence on the first test. Again, the proportions of shifts from the lower confidence categories contrast with the overall proportion of .908 for the highest confidence rating on the second trial of a $\mathrm{C}-\mathrm{C}$ pair. It should be noted, however, that this function is at least partly determined by the probability of making a large shift in confidence. This is reflected in the fact that for the other three cases, i.e., $\mathrm{N}-\mathrm{C}, \mathrm{C}-\mathrm{N}, \mathrm{N}-\mathrm{N}$, the proportion of highest confidence ratings on the second trial also appears to be a slightly increasing function of the first trial confidence. In none of these three cases, however, are the increases as large or stable as for $\mathrm{C}-\mathrm{C}$. An analysis of variance on mean confidence ratings across trials for responses correct on all three trials indicated a highly significant increase $(\mathrm{p}<.001)$.

\section{Discussion}

The major results are in agreement with the hypothesis that the confidence rating is a reflection of the strength of the learned association between stimulus and response. They also suggest that confidence ratings may be used to greatly increase the information yield from verbal learning experiments. Furthermore, the demonstration that confidence increases for correct responses following correct responses on a previous trial, is difficult to reconcile with all-or-none learning theory. The simplicity of the learning situation should have been highly reflective for an all-or-none learning process (Underwood \& Keppel, 1962), and yet our results suggest a continuum of states of association between stimuli and responses rather than just learned and unlearned.

\section{References}

Egan, J. P. Recognition memory and the operating characteristic. Indiana University Hearing and Communication Laboratory. Tech. Note AFCRC - TN - 58-51, 1958.

Egan, J. P., Schulman, A. I., \& Greenberg, G. Z. Operating characteristics determined by binary decisions and by ratings. $J$. Acoust. Soc. Amer., 1959, 31, 768-773.

Murdock, B. B., Jr. Signal-detection theory and short term memory. J. exp. Psychol., 1965, 70, 443-447.

Swets, J. A., Tanner, W. P., Jr., \& Birdsall, T. G. Decision processes in perception. Psychol. Rev., 1961, 68, 301-340.

Underwood, B. J., \& Keppel, G. One trial learning. J. verbal Learn. verbal Behav., 1962, 1, 1-13.

Note

1. This research was supported by N. R. C. Grant No. APA 160. 